Original article

\title{
Determinants or barriers associated with specific routine check-up in antenatal care in gestational period: A study from EAG states, India
}

\author{
Madhurima Sarkar **, Tanu Das *, Tamal Basu Roy ${ }^{* * *}$ \\ Department of Geography, Raiganj University, Raiganj, Uttar Dinajpur District, West Bengal, 733134, India
}

\section{A R T I C L E I N F O}

\section{Keywords:}

Antenatal care

Routine check-up

Maternal health

EAG states

NFHS

Gestational period

\begin{abstract}
A B S T R A C T
Background: For a long period of time Empowered Action Group States (EAG) of India are cursed with maternal mortality and morbidity. Most of the pregnant women are not acquiescent to receive the routine check-up during Antenatal Care (ANC) and many of them cautiously skip the same. The study aims to explore the obstacle of ANC in women's perspective and their participation in this.

Methods: The study has incorporated most recent fourth round of National Family Health Survey data conducted in 2015-16. Descriptive statistics along with association test and binary logistic regression model have been applied to understand the differential and determinants of ANC.

Result: The behaviour of not taking ANC check-ups (measuring the weight and BP, tested urine and blood sample, taking iron supplements) during pregnancy was significantly higher among women who married in early age ( $<18$ years), had no formal education, belonged to poor socioeconomic status.

Conclusion: Most of the maternal health issues during the gestational period are associated with socio-economic and demographic aspects and mostly due to unawareness and ignorance of the people. It would be benevolent in increasing awareness and taking the necessary visit to receive the routine check-up in ANC is for the duo mothers and foetus.
\end{abstract}

\section{Introduction}

Maternal health always remains one of the priorities of global concern, mostly in developing and under developed countries. In fact, improving maternal health was one of the major priorities of Millennium Development Goal (MDG). Although, still 99\% maternal death occurs in less developed countries with low resource settings. ${ }^{1}$ In India every year, approximately 28 million women experiences pregnancy, out of these an estimated 1.3 lakhs maternal fatality occurs. ${ }^{2}$ India has made remarkable progress in maternal health outcome over last three decades but still high maternal mortality persists across empowered action group (EAG) states. ${ }^{3}$ India accounts one fifth of maternal mortality worldwide. ${ }^{4}$ Most of the maternal health problem in EAG states continues to be associated with nutrition, socioeconomic marginalisation, poverty and mostly the unawareness and ignorance of the people..$^{5}$ Majority of people of these states has poor knowledge regarding importance of receiving Antenatal Care (ANC), Delivery Care (DC) and Postnatal Care
(PNC). ${ }^{6}$ ANC foster physical and psychological health, welfare of women and prepare them for safe birth. ${ }^{7}$ ANC detects many complications occurring before childbirth and considerably look after the improvement of the health of mother and her child. ${ }^{8,9}$ It includes various diagnostics procedures, vaccination, counselling on nutrition and regular screening of pregnant women which can prevent early complexities and treat conditions that may threaten the health of mother along with foetus. ${ }^{10}$ It can detect maternal infections that are dangerous to the infant and other problems like-anaemia, hypertension, pre-eclampsia, vaginitis, urinary tract infections, HIV complications, malnutrition, poor foetal growth etc. ${ }^{11}$

During gestation period the first visit of ANC consists of weight measurement, blood pressure (BP) measurement, blood test and urine test for the pregnant women, so the attending the first antenatal care visit is very crucial for pregnant women for improving health assessment. ${ }^{12,13}$ Weight measurement during maternity care is very important because it estimates the risk during pregnancy and customised growth

\footnotetext{
Abbreviations: MMR, Maternal Mortality Rate; ANC, Antenatal Care; BP, Blood Pressure; NFHS, National Family Health Survey.

* Corresponding author.

** Corresponding author.

$* * *$ Corresponding author.

E-mail addresses: madhurima.sarkar21@gmail.com (M. Sarkar), tanudas.04321@gmail.com (T. Das), raiganjgeo@gmail.com (T.B. Roy).
} 
chart is calculated to assess the baby's progress. Routine measurement of weight of the pregnant women can assess excess weight gain or poor weight gain. Sometimes excess weight gain may cause pre-eclampsia. ${ }^{14}$ Eclampsia and pre-eclampsia, oedema, obesity, poly hydramnios etc. are the serious problem due to weight gain during pregnancy, causes maternal, fetal and neonatal illness and mortality. ${ }^{15} \mathrm{BP}$ (Blood Pressure) examining is the most crucial diagnosis; frequently screening test is required during antenatal period to manage hypertensive disorder. ${ }^{16,17}$ Chronic hypertension analogous with adverse maternal outcome, it may be controlled by maintaining maternal BP and proper observation of foetal growth. ${ }^{18}$ Blood test is required in early pregnancy to examine haemoglobin level, ${ }^{19} \mathrm{Rh}$ factor, blood group, iron deficiency and anaemia, ${ }^{20}$ rubella immunity, syphilis, HIV, hepatitis B and hepatitis C. ${ }^{21}$ Routine urine test is also mandatory in each trimester know urinary tract infection, protein level and sugar in urine, to avoid pregnancy related complications that can push the health of mother and infant at risk. ${ }^{22}$ Precautions of Iron tablets or syrup and folic acid supplements are recommended for pregnant women to prevent anaemia, preterm birth, low birth weight, puerperal sepsis. ${ }^{23}$ The distinguished socio-economic and demographic characters either facilitate or create obstacles on the way of receiving the routine check-up care and treatments during antenatal care in EAG states in India. ${ }^{25}$ The recognized major socio-economic and demographic determinants might implicate ultimately on the response on desired ANC visit and create uneven health outcomes and increased incidence of maternal illness and mortality. ${ }^{26,27}$

Govt. of India has prepared a list of eight states namely Empowered Action Groups or EAG states which are less developed or very poor in socio-economic as well as demographic index as compared to other states of India. EAG states comprise of Bihar, Jharkhand, Chhattisgarh, Madhya Pradesh, Orissa, Rajasthan, Uttarakhand and Uttar Pradesh. ${ }^{28}$ These states are among the lower-middle income economics where women are less privileged in respect of food, medical facilities and a large proportion of its population still living in poverty. ${ }^{29}$ EAG states consists of $45 \%$ of India's population and lagging far behind in health and socio-economic dimension. ${ }^{30}$ The percentage of Maternal Mortality Rate (MMR) is very high (61.6\%) in these states. ${ }^{31}$ Better access, qualitative reproductive health care to improve maternal health of the women and to reduce MMR is the need of hour. ${ }^{32}$ The study gives its focus on the treatments provided to the pregnant women during ANC visit and to recognize the major non proximate determinants and its association with the proper utilisation of ANC treatments. The participation of pregnant women in utilisation of ANC treatments is largely influenced by their individual socio-economic background. ${ }^{33}$ Most of the women are not willing to receive the routine check-up embedded within the ANC and many of them deliberately skip the same. ${ }^{34}$ Earlier little study has been conducted on EAG states that investigate the determinant factors and its individual relevance on participation in receiving the desired ANC care. The prime objective of this study is to recognize the major determinants, its importance in receiving the various individual antenatal routine check-up care usages about mother in EAG states of India.

\section{Materials and methods}

\subsection{Data source}

The data for the research presented here come from the fourth round of the National Family Health Survey (NFHS-4), conducted in 2015-2016. This cross sectional, nationally representative survey was carried out under the guidance of the Ministry of Health and Family Welfare (MoHFW), Govt. of India and International Institute for Population Sciences (IIPS), Mumbai. Two stage stratified sampling design was adopted to select the sample. This face to face $\&$ door to door sample survey collects information for 601,509 households, 6, 99,686 women aged 15-49 years with a response rate of $97 \%$, and 112,122 men aged $15-54$ years with a response rate of $92 \%$. The common objectives of this survey were to obtain reliable data relating to fertility and family planning, utilisation of maternal and child health care treatments, infant and child mortality, nutrition, women empowerment, domestic violence and knowledge on HIV/AIDS etc.

\subsection{Ethical statement}

The study is based on a secondary data set from the recent NFHS-4 survey with no identifiable information on the survey participants. NFHS- 4 obtained the consent before and during the survey. This dataset is available in the public domain for research use and, hence, no ethical approval was needed specifically for the present study. The data can be freely accessed from the DHS website at https://www.dhsprogram.com /data.

\subsection{Study participants}

This current study used women data file and total 1, 47,049 sample women of reproductive age (15-49 Years) group were interviewed for EAG states. However, after removing all the missing cases, and checking outlier, homogeneity, multicollinearity the final estimation was carried out based on 80,173 sample women of reproductive age group in EAG states. The married women who were pregnant at least once in her life time and taking the essential antenatal care treatments at least once are included in this present study. The study excluded all the pregnant who do not take any antenatal care treatments from any medical professionals and who are currently pregnant during the survey. The count variables were categorized and categorized variables were recategorized based on study requirements.

\subsection{Methodology adopted}

To examining the determinants or barriers in receiving various antenatal care treatments initially, a non-parametric chi-square test statistic was performed at level $\mathrm{P}<0.05$ to determine the associations between respondent's individual characteristics and various essential antenatal care treatments (During pregnancy - weight measured, BP measured, Urine test, Blood test, Iron supplement taken). After that multivariate binary logistic regression model, including all potential determinants or barriers in receiving various antenatal care treatments was estimated to arrive at individually adjusted estimates. This likelihood ratio statistics can help to compare each category with reference to a particular category. Before going to any inferential statistics some descriptive measures have been performed such as frequency distribution, weighted percentage distribution, cross tabulation etc. to get an idea about the maternal (respondent's) background characteristics. Percentage distribution shown in this present work is weighted percentage but the numbers of samples $(\mathrm{N})$ are actual number. All the statistical analysis was performed through IBM SPSS statistics software 25.

\subsection{Outcome variables}

The key outcome variables of interest in this present study were various essential ANC treatment not receiving behaviour during the gestational (from the last menstruation to 42 weeks) period of pregnant women. As per previous literatures it is very important for pregnant women to measure her weight, checking BP, test urine \& blood sample and to take iron supplements to maintain her pregnancy related health. ${ }^{21-23}$ All of these essential ANC treatments were considered as outcome variables and the variables are dichotomize in nature, which mean if the respondent gives negative response, it coded as 1 and the affirmative response were coded as 0 . This study was limited to those women who were pregnant at least once in her life course and take at least once ANC treatments from medical professionals. 


\subsection{Exposure variables}

Based on the existing literature review some individual level characteristics and family level characteristics were considered as the exposure variables for this present study. The individual level characteristics were as follows - a. Women age (Categorized as $<20$ Years, 20-30 Years, $>30$ Years) b. Women education (Categorized as No education, Primary, Secondary, Higher) c. Age at first marriage (Categorized as $<18$ Years, 18-25 Years, 26-30 Years and $>30$ Years) d. Number of taken antenatal care treatments (Categorized as 1 to 3 times, 4 to 5 times and $>5$ times). Family level characteristics were including e. Place of residence (Dichotomized as Rural or Urban) f. Wealth quintile (Categorized as Poorest, Poorer, Middle, Richer, Richest) g. Religious belief (Categorized as Hindu, Muslim and Others) and h. Affiliation to a social category (Categorized as Schedule Caste [SC], Scheduled Tribe [ST] and Others). Wealth index is a proxy indicator of household's socioeconomic status. Household wealth quintile has been measured from the ownership of household assets including consumer items and dwelling characteristics. A score was generated for each individual using principal component analysis and categorized into five quintiles each represents $20 \%$ of the respondents, between 1 (poorest) and 5 (richest).

\section{Results}

\subsection{Socio-demographic characteristics of the study participants}

The present study included only reproductive woman whose age ranged from 15 years to 49 years, out of which more than $75 \%$ women were measured their weight (80.10\%) and blood pressure (78.80\%), test their urine sample (77.20\%) and blood sample (75.80\%) and also taken iron supplements (76.40\%) under the guidance of any medical professionals during their gestational period. Table 1 shows majority of the participants 62,004 that is more the three of fourth $(76.90 \%)$ were between the age group $20-30$ years. Nearly $40 \%$ (39.20\%) women were not attended in formal education and $38.80 \%$ women married before completing their legal age of marriage that is 18 years. Most of the women were rural $(77.20 \%)$ resident and belonged in middle to poorest (71.5\%) socioeconomic status. This study also showed that majority of the respondent $(84.70 \%)$ believed in Hinduism and only $1 \%$ women were apart from any socially reserved (SC/ST) category. Current estimate also revealed that total 45,289 women $(58.40 \%)$ in this present study were attained more than 3 antenatal care visits.

\subsection{Behavioural characteristics of women in receiving various antenatal care treatments}

Table 2 Shows that the women whose age were more than 30 years were comparatively less aware for taking various antenatal care related treatment such as weight measurement, BP measure, urine sample test, blood sample test and in taking iron supplements. Cross tabulation clearly indicates that the proportion in not taking various essential ANC treatments were higher in those women who had no formal education as well as married before completing the legal age that is 18 years. Very minimal proportion of women who resides in rural areas are mostly aware about their pregnancy health as compared to urban women. In this present estimation it is noticed that $24 \%$ rural pregnant women do not measure their BP, $25.6 \%$ pregnant women do not test their urine sample and $72.5 \%$ women do not test their blood sample in their gestational period. A noticeable proportion of women who were belonged to poorer and poorest socioeconomic status were far from receiving routine ANC treatments. The proportion in not taking ANC treatments were higher in those women who believed in Muslim religious belief and affiliated to socially reserved (SC/ST) category. It is perhaps clear from the background characteristics and the recent estimates that the women who visited more than 5 times for ANC, they have received a lot of these treatments as compared to the women who visited
Table: 1

Background characteristics \& weighted percentage distribution of reproductive age group women in EAG state who were received ANC treatments.

\begin{tabular}{|c|c|c|}
\hline Background Characteristics & Number (N) & Weighted Percentage \\
\hline \multicolumn{3}{|l|}{ Weight Measured } \\
\hline Yes & 67118 & 80.10 \\
\hline No & 13055 & 19.90 \\
\hline \multicolumn{3}{|l|}{ BP Measured } \\
\hline Yes & 65222 & 78.80 \\
\hline No & 14951 & 21.20 \\
\hline \multicolumn{3}{|l|}{ Get urine tested } \\
\hline Yes & 63233 & 77.20 \\
\hline No & 16940 & 22.80 \\
\hline \multicolumn{3}{|l|}{ Blood tested } \\
\hline Yes & 62666 & 75.80 \\
\hline No & 17507 & 24.20 \\
\hline \multicolumn{3}{|l|}{ Iron Supplements taken } \\
\hline Yes & 62891 & 76.40 \\
\hline No & 17096 & 23.40 \\
\hline Don't Know/Missing & 186 & 00.20 \\
\hline \multicolumn{3}{|l|}{ Women's Age } \\
\hline$<20$ Years & 2286 & 02.90 \\
\hline 20-30 Years & 62004 & 76.90 \\
\hline$>30$ Years & 15883 & 20.20 \\
\hline \multicolumn{3}{|l|}{ Women's Education } \\
\hline No education & 30606 & 39.20 \\
\hline Primary & 34816 & 42.00 \\
\hline Secondary & 6153 & 07.70 \\
\hline Higher & 8598 & 11.10 \\
\hline \multicolumn{3}{|l|}{ Age at First Marriage } \\
\hline$<18$ Years & 30591 & 38.80 \\
\hline 18-25 Years & 46988 & 58.10 \\
\hline 26-30 Years & 2303 & 02.80 \\
\hline$>30$ Years & 291 & 00.40 \\
\hline \multicolumn{3}{|l|}{ Place of Residence } \\
\hline Rural & 60989 & 77.20 \\
\hline Urban & 19184 & 22.80 \\
\hline \multicolumn{3}{|l|}{ Wealth Quintile } \\
\hline Poorest & 23379 & 29.70 \\
\hline Poorer & 18887 & 23.70 \\
\hline Middle & 14639 & 18.10 \\
\hline Richer & 12128 & 14.90 \\
\hline Richest & 11140 & 13.60 \\
\hline \multicolumn{3}{|l|}{ Religion } \\
\hline Hindu & 68103 & 84.70 \\
\hline Muslim & 10242 & 13.70 \\
\hline Others & 1828 & 01.60 \\
\hline \multicolumn{3}{|l|}{ Category } \\
\hline SC & 70350 & 90.70 \\
\hline ST & 8947 & 08.30 \\
\hline Others & 876 & 01.00 \\
\hline \multicolumn{3}{|l|}{ ANC Visit } \\
\hline 1 to 3 & 45289 & 58.40 \\
\hline 4 to 5 & 19621 & 23.50 \\
\hline$>5$ & 15263 & 18.10 \\
\hline
\end{tabular}

one to three times.

\subsection{Test of significance between receiving various ANC treatments by the recipient and their background characteristics}

Table 3 shows the bivariate association along with effect size through Chi-square test between selected dependent and independent variables. All most all the essential ANC treatments (During pregnancy weight measurement, checking BP, blood sample and urine sample test, taking iron supplement etc.) are significantly associated (at level P $<0.05$ ) with respondent's personal and family level characteristics but the effect size is different for respondent's each individual level and family level characteristics. The effect of each independent variable on selected dependent variable is measured by Phi coefficient and Cramer's V approach, which are nothing but one kind of correlation coefficient and value can range from 0 to 1 , with higher value indicating stronger association between the variables. Present estimation (Table 3 ) indicated that respondent's current age, education, age at marriage and wealth 
Table: 2

Utilisation of ANC treatments by study population according to their background characteristics in EAG states, India.

\begin{tabular}{|c|c|c|c|c|c|c|c|c|c|c|}
\hline \multirow[t]{3}{*}{ Background Characteristics } & \multicolumn{10}{|c|}{ Various ANC Treatments During Gestational Period } \\
\hline & \multicolumn{2}{|c|}{ Weight Measured (\%) } & \multicolumn{2}{|c|}{ BP Measured (\%) } & \multicolumn{2}{|c|}{ Urine Sample Tested (\%) } & \multicolumn{2}{|c|}{ Blood Sample Tested (\%) } & \multicolumn{2}{|c|}{ Iron Supplements Taken (\%) } \\
\hline & Yes & No & Yes & No & Yes & No & Yes & No & Yes & No \\
\hline \multicolumn{11}{|l|}{ Women's Age } \\
\hline$<20$ Years & 82.2 & 17.8 & 80.6 & 19.4 & 78.9 & 21.1 & 79.2 & 20.8 & 76.2 & 23.8 \\
\hline 20-30 Years & 82.0 & 18.0 & 80.1 & 19.9 & 78.5 & 21.5 & 77.4 & 22.6 & 77.2 & 22.8 \\
\hline$>30$ Years & 76.4 & 23.6 & 73.6 & 26.4 & 71.9 & 28.1 & 69.2 & 30.8 & 74.4 & 25.6 \\
\hline \multicolumn{11}{|l|}{ Women's Education } \\
\hline No education & 73.8 & 26.2 & 69.5 & 30.5 & 67.4 & 32.6 & 64.3 & 35.7 & 69.7 & 30.3 \\
\hline Primary & 83.8 & 16.2 & 82.6 & 17.4 & 81.2 & 18.8 & 80.6 & 19.4 & 78.7 & 21.3 \\
\hline Secondary & 86.0 & 14.0 & 86.0 & 14.0 & 85.8 & 14.2 & 85.1 & 14.9 & 81.6 & 18.4 \\
\hline Higher & 91.1 & 08.9 & 92.4 & 07.6 & 90.9 & 9.1 & 91.6 & 08.4 & 87.5 & 12.5 \\
\hline \multicolumn{11}{|l|}{ Age at First Marriage } \\
\hline$<18$ Years & 77.6 & 22.4 & 74 & 26 & 72.6 & 27.4 & 70.1 & 29.9 & 73.1 & 26.9 \\
\hline 18-25 Years & 82.6 & 17.4 & 81.4 & 18.6 & 79.7 & 20.3 & 78.9 & 21.1 & 78.2 & 21.8 \\
\hline 26-30 Years & 89.8 & 10.2 & 89.7 & 10.3 & 88.4 & 11.6 & 87.6 & 12.4 & 83.4 & 16.6 \\
\hline$>30$ Years & 88.7 & 11.3 & 88.3 & 11.7 & 84.6 & 15.4 & 84.6 & 15.4 & 82.1 & 17.9 \\
\hline \multicolumn{11}{|l|}{ Place of Residence } \\
\hline Rural & 79.1 & 20.9 & 76.0 & 24.0 & 74.4 & 25.6 & 27.5 & 72.5 & 75.5 & 24.5 \\
\hline Urban & 86.8 & 13.2 & 88.2 & 11.8 & 86.7 & 13.3 & 13.1 & 86.9 & 80.4 & 19.6 \\
\hline \multicolumn{11}{|l|}{ Wealth Quintile } \\
\hline Poorest & 75.0 & 25.0 & 68.7 & 31.3 & 66.4 & 33.6 & 63.1 & 36.9 & 72.8 & 27.2 \\
\hline Poorer & 77.5 & 22.5 & 75.1 & 24.9 & 74.5 & 25.5 & 72.7 & 27.3 & 74.6 & 25.4 \\
\hline Middle & 81.9 & 18.1 & 81.0 & 19.0 & 80.2 & 19.8 & 78.9 & 21.1 & 76.5 & 23.5 \\
\hline Richer & 86.0 & 14.0 & 87.7 & 12.3 & 85.8 & 14.2 & 86.2 & 13.8 & 79.4 & 20.6 \\
\hline Richest & 92.7 & 07.3 & 94.6 & 05.4 & 92.2 & 07.8 & 93.3 & 06.7 & 84.8 & 15.2 \\
\hline \multicolumn{11}{|l|}{ Religion } \\
\hline Hindu & 82.0 & 18.0 & 79.3 & 20.7 & 78.0 & 22.0 & 76.7 & 23.3 & 77.7 & 22.3 \\
\hline Muslim & 72.5 & 27.5 & 74.3 & 25.7 & 71.8 & 28.2 & 69.6 & 30.4 & 67.3 & 32.7 \\
\hline Others & 93.6 & 06.4 & 89.1 & 10.9 & 81.7 & 18.3 & 81.2 & 18.8 & 85.4 & 14.6 \\
\hline \multicolumn{11}{|l|}{ Category } \\
\hline SC & 79.7 & 20.3 & 78 & 22.0 & 76.8 & 23.2 & 75.2 & 24.8 & 75.5 & 24.5 \\
\hline ST & 92.9 & 07.1 & 87.0 & 13.0 & 80.9 & 19.1 & 81.7 & 18.3 & 85.7 & 14.3 \\
\hline Others & 85.6 & 14.4 & 83.7 & 16.3 & 82.6 & 17.4 & 80.7 & 19.3 & 80.4 & 19.6 \\
\hline \multicolumn{11}{|l|}{ ANC Visit } \\
\hline 1 to 3 & 73.7 & 26.3 & 69.7 & 30.3 & 67.9 & 32.1 & 65.5 & 34.5 & 70.8 & 29.2 \\
\hline 4 to 5 & 89.2 & 10.8 & 89.5 & 10.5 & 88.1 & 11.9 & 88.2 & 11.8 & 82.9 & 17.1 \\
\hline$>5$ & 93.3 & 06.7 & 94.1 & 05.9 & 93.2 & 06.8 & 93.0 & 07.0 & 86.0 & 14.0 \\
\hline
\end{tabular}

Table: 3

Chi-square associations between maternal background characteristics and different antenatal care treatments during gestational period in EAG state.

\begin{tabular}{|c|c|c|c|c|c|c|c|c|c|c|c|c|c|c|c|}
\hline \multirow{3}{*}{$\begin{array}{l}\text { Background } \\
\text { Characteristics }\end{array}$} & \multicolumn{15}{|c|}{ Antenatal Care Treatments During Gestational Period } \\
\hline & \multicolumn{3}{|c|}{ Weight Measured } & \multicolumn{3}{|c|}{ BP Measured } & \multicolumn{3}{|c|}{ Urine sample Tested } & \multicolumn{3}{|c|}{ Blood Sample Tested } & \multicolumn{3}{|c|}{ Iron Supplements Taken } \\
\hline & $\begin{array}{l}\chi^{2}- \\
\text { Value }\end{array}$ & $\begin{array}{l}\text { P- } \\
\text { Value }\end{array}$ & $\begin{array}{l}\text { Effect } \\
\text { Size }\end{array}$ & $\begin{array}{l}\chi^{2}- \\
\text { Value }\end{array}$ & $\begin{array}{l}\text { P- } \\
\text { Value }\end{array}$ & $\begin{array}{l}\text { Effect } \\
\text { Size }\end{array}$ & $\begin{array}{l}\chi^{2}- \\
\text { Value }\end{array}$ & $\begin{array}{l}\text { P- } \\
\text { Value }\end{array}$ & $\begin{array}{l}\text { Effect } \\
\text { Size }\end{array}$ & $\begin{array}{l}\chi^{2}- \\
\text { Value }\end{array}$ & $\begin{array}{l}\mathrm{P}- \\
\text { Value }\end{array}$ & $\begin{array}{l}\text { Effect } \\
\text { Size }\end{array}$ & $\begin{array}{l}\chi^{2}- \\
\text { Value }\end{array}$ & $\begin{array}{l}\mathrm{P}- \\
\text { Value }\end{array}$ & $\begin{array}{l}\text { Effect } \\
\text { Size }\end{array}$ \\
\hline Women's Age & 212.7 & 0.000 & 0.6 & 266.5 & 0.000 & 0.5 & 249.5 & 0.000 & 0.5 & 396.8 & 0.000 & 0.6 & 60.8 & 0.000 & 0.7 \\
\hline $\begin{array}{l}\text { Women's } \\
\text { Education }\end{array}$ & 1640.2 & 0.000 & 0.8 & 2703.5 & 0.000 & 0.6 & 2822.6 & 0.000 & 0.5 & 3603.5 & 0.000 & 0.7 & 1376.7 & 0.000 & 0.5 \\
\hline $\begin{array}{l}\text { Age at First } \\
\text { Marriage }\end{array}$ & 442.5 & 0.000 & 0.5 & 266.5 & 0.000 & 0.5 & 738.6 & 0.000 & 0.4 & 972.3 & 0.000 & 0.4 & 360.5 & 0.000 & 0.6 \\
\hline $\begin{array}{l}\text { Place of } \\
\text { Residence }\end{array}$ & 330.4 & 0.000 & 0.3 & 968.7 & 0.000 & 0.2 & 1044.0 & 0.000 & 0.1 & 1357.9 & 0.000 & 0.2 & 138.2 & 0.000 & 0.1 \\
\hline Wealth Quintile & 1276.4 & 0.000 & 0.7 & 3015.4 & 0.000 & 0.6 & 3058.5 & 0.000 & 0.5 & 3980.1 & 0.000 & 0.5 & 537.6 & 0.000 & 0.6 \\
\hline Religion & 760.7 & 0.000 & 0.3 & 234.3 & 0.000 & 0.3 & 176.3 & 0.000 & 0.3 & 240.6 & 0.000 & 0.2 & 671.6 & 0.000 & 0.1 \\
\hline Category & 783.2 & 0.000 & 0.2 & 316.1 & 0.000 & 0.3 & 60.1 & 0.000 & 0.2 & 145.6 & 0.000 & 0.3 & 429.2 & 0.000 & 0.2 \\
\hline ANC Visit & 3569.7 & 0.000 & 0.7 & 5275.3 & 0.000 & 0.6 & 5551.7 & 0.000 & 0.6 & 6306.6 & 0.000 & 0.7 & 2148.5 & 0.000 & 0.5 \\
\hline
\end{tabular}

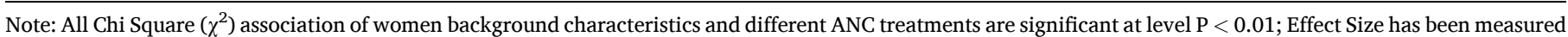
through Phi coefficient and Cramer's V approach.

quintile has stronger effect and significant association with receiving every essential ANC service during their gestational period. On the other hand, women's religious belief, affiliation to a particular social category and their living place has little effect on their behavioural aspects in receiving various ANC treatments during the gestational period. ANC visit to a medical professional during gestational period is not a respondent's personal or family level characteristics rather than one can say it is a societal behavioural characteristic for pregnant women. Result shows there is a significant association between respondent's individual level and family level characteristics and their response in antenatal check-up, such as during pregnancy weight measurement $\left(\chi^{2}: 3569.7 ; \mathrm{P}\right.$ : $<0.001$; Cramer's V: 0.7), checking blood pressure $\left(\chi^{2}: 5275.3\right.$; P: $<$ 0.001; Cramer's V: 0.6), urine sample test $\left(\chi^{2}: 5551.7\right.$; P: $<0.001$; Cramer's V: 0.6), blood sample test $\left(\chi^{2}: 6306.6 ; \mathrm{P}:<0.001\right.$; Cramer's V: $0.7)$ and iron supplement taken $\left(\chi^{2}: 2148.5 ; \mathrm{P}:<0.001\right.$; Cramer'sV:0.5). 
3.4. Test of association along with likelihood ratio between not utilisation of ANC treatments by the recipients and their background characteristics

Table 4 represents the adjusted association along with likelihood ratio for recipient's various antenatal cares (ANC) related treatment not taking behaviour in their gestational period at each individual level \& family level characteristic. Logistic regression for each (Do not weight measured/Do not BP measures/Do not urine sample test/Do not blood sample test/Do not iron supplements taken) antenatal care related treatment not taking behaviour and its background characteristics were performed separately but Table 4 represent here is a combined view.

\subsubsection{Barriers in weight measurement}

During pregnancy the behaviour of not taking ANC treatment for example weight measurement by the women is statistically and significantly associated with all most all the chosen maternal individual level and family level characteristics. The odds of women's not weight measurement behaviour during pregnancy was significantly higher (AOR: 1.44; P < 0.001; CI: 1.38-1.62) among those women whose current age were less than 20 years compared to those women who were more than 30 years old. Result revealed that the likelihood for not weight measurement during pregnancy was significantly higher among those women, who had no formal education (AOR: 1.59; P $<0.001$; CI: 1.54-1.65), married before completing their age of 18 years (AOR: 1.46; $\mathrm{P}<0.001$; CI: 1.29-1.58), resided at rural environment (AOR: 1.64; P < 0.001; CI: 1.61-1.67), belonged to poor socio economic status, believed in Muslim religion (AOR: 1.53; P $<0.001$; CI: 1.27-1.56), affiliated to socially reserved category [SC(AOR: 1.21; P < 0.001; CI: 1.13-1.45) ST (AOR: 1.33; P $<0.001$; CI: 1.23-1.51)] and attain less than 3 antenatal care visit (AOR: 1.25; P < 0.001; CI: 1.23-1.27) to a medical professional compared to those women who had higher education, married after 30 years of age, resides in urban environment, belonged to upper socioeconomic status, believed in other religious belief rather than Hindu \& Muslim, affiliated to general category and attain more than 5 ANC visit to the medical professionals.

\subsubsection{Barriers in blood pressure (BP) measurement, urine sample test, blood sample test and taking iron supplements}

The study estimation (Table 4) depicts that all the behaviour of not taking ANC treatments (not measuring the BP, not tested urine sample, not tested blood sample, not taking iron supplements) was significantly higher among those women whose age was less than 20 years, had no formal education, got married before completing their age 18, resides in rural environment, belonged to poor socioeconomic status, believed in Muslim religion, affiliated to socially reserved category and took at least 1 to 3 ANC visit to the medical professional compared to those women who had higher education, married after 30 years of age, resides in urban environment, belonged to upper socioeconomic status, believed in other religious belief rather than Hindu \& Muslim, affiliated to general category and attain more than 5 ANC visit to a medical professionals. Table 4 delineates women having less than 20 years of age were (AOR 1.6; P < 0.001; CI: 1.43-1.84) times less likely to measured their BP and were(AOR: 1.27; P < 0.001; CI: 1.11-1.48) times less likely to tested their blood sample as compared to women aged more than 30

Table: 4

Adjusted odd ratios for not taking various ANC treatments by the recipients at each individual and family level characteristic in gestational period.

\begin{tabular}{|c|c|c|c|c|c|c|c|c|c|c|}
\hline \multirow{3}{*}{ Background Characteristics } & \multicolumn{10}{|c|}{ Behaviour of Not Taking ANC Treatments During Gestational Period } \\
\hline & \multicolumn{2}{|c|}{$\begin{array}{l}\text { Did not Weight } \\
\text { Measured }\end{array}$} & \multicolumn{2}{|c|}{ Did not BP Measured } & \multicolumn{2}{|c|}{$\begin{array}{l}\text { Did not Urine sample } \\
\text { Tested }\end{array}$} & \multicolumn{2}{|c|}{$\begin{array}{l}\text { Did not Blood Sample } \\
\text { Tested }\end{array}$} & \multicolumn{2}{|c|}{$\begin{array}{l}\text { Did not Iron Supplements } \\
\text { Taken }\end{array}$} \\
\hline & AOR & $95 \% \mathrm{CI}$ & AOR & $95 \% \mathrm{CI}$ & AOR & $95 \% \mathrm{CI}$ & AOR & $95 \% \mathrm{CI}$ & AOR & $95 \% \mathrm{CI}$ \\
\hline \multicolumn{11}{|l|}{ Women's Age } \\
\hline$<20$ Years & 1.44 & $1.38-1.62$ & 1.63 & $1.43-1.84$ & 1.49 & $1.32-1.67$ & 1.27 & $1.11-1.48$ & 1.57 & $1.48-1.83$ \\
\hline $\begin{array}{l}\text { 20-30 Years } \\
>30 \text { Years (Ref.) }\end{array}$ & .29 & $.22-.35$ & .30 & $.24-.36$ & .86 & $.51-.92$ & .36 & $.30-.42$ & .37 & $.30-.42$ \\
\hline \multicolumn{11}{|l|}{ Women's Education } \\
\hline No education & 1.59 & $1.54-1.65$ & 1.55 & $1.49-1.61$ & 1.55 & $1.51-1.61$ & 1.52 & $1.47-1.57$ & 1.57 & $1.49-1.68$ \\
\hline Primary & 1.09 & $1.00-1.13$ & 1.08 & $1.00-1.12$ & 1.07 & $1.00-1.11$ & 1.11 & $1.08-1.22$ & 1.05 & $0.90-1.23$ \\
\hline Secondary & 0.80 & $.71-.90$ & 0.77 & $.68-.87$ & 0.84 & $.75-.94$ & 0.79 & $.71-.89$ & 0.77 & $.71-.91$ \\
\hline \multicolumn{11}{|l|}{ Higher (Ref.) } \\
\hline \multicolumn{11}{|l|}{ Age at First Marriage } \\
\hline$<18$ Years & 1.46 & $1.29-1.58$ & 1.48 & $1.32-1.74$ & 1.61 & $1.42-1.88$ & 1.49 & $1.34-.173$ & 1.38 & $1.2-1.51$ \\
\hline 18-25 Years & 0.32 & $.27-.41$ & 0.57 & $.38-.88$ & 0.71 & $.49-.93$ & 0.60 & $.41-.88$ & 0.52 & $.48-.63$ \\
\hline 26-30 Years & 0.27 & $.21-.42$ & 0.92 & $.79-1.21$ & 0.84 & $.76-.97$ & .60 & $.38-.69$ & 0.79 & $.59-.91$ \\
\hline \multicolumn{11}{|l|}{$>30$ Years (Ref.) } \\
\hline \multicolumn{11}{|l|}{ Place of Residence } \\
\hline Rural & 1.64 & $1.61-1.67$ & 1.84 & $1.79-1.89$ & 1.13 & $1.01-1.26$ & 1.27 & $1.21-1.36$ & 1.21 & $1.99-1.33$ \\
\hline \multicolumn{11}{|l|}{ Urban(Ref.) } \\
\hline \multicolumn{11}{|l|}{ Wealth Quintile } \\
\hline Poorest & 1.28 & $1.26-1.30$ & 1.29 & $1.27-1.33$ & 1.40 & $1.37-1.44$ & 1.30 & $1.27-1.33$ & 1.26 & $1.19-1.37$ \\
\hline Poorer & 1.30 & $1.28-1.33$ & 1.35 & $1.32-1.39$ & 1.51 & $1.47-1.57$ & 1.40 & $1.36-1.44$ & 1.31 & $1.29-1.36$ \\
\hline Middle & 0.37 & $.34-.41$ & 0.42 & $.39-.48$ & 0.60 & $.55-.66$ & 0.47 & $.43-.52$ & 0.37 & $.27-.39$ \\
\hline Richer & 0.50 & $.46-.67$ & 0.60 & $.55-.68$ & 0.74 & $.68-.82$ & 0.64 & $.58-.71$ & 0.50 & $.48-.71$ \\
\hline \multicolumn{11}{|l|}{ Richest (Ref.) } \\
\hline \multicolumn{11}{|l|}{ Religion } \\
\hline Hindu & 0.36 & $.21-.41$ & 0.68 & $.58-.81$ & 0.49 & $0.42-.86$ & 0.87 & $.66-1.11$ & 0.62 & $.51-.67$ \\
\hline Muslim & 1.53 & $1.27-1.56$ & 1.50 & $1.43-1.61$ & 1.69 & $1.60-1.80$ & 1.26 & $1.14-1.34$ & 1.09 & $1.02-1.14$ \\
\hline \multicolumn{11}{|l|}{ Others (Ref.) } \\
\hline \multicolumn{11}{|l|}{ Social Category } \\
\hline SC & 1.21 & $1.13-1.45$ & 1.23 & $1.17-1.29$ & 1.27 & $1.15-1.43$ & 1.19 & $1.11-1.42$ & 1.27 & $1.25-1.69$ \\
\hline ST & 1.33 & $1.23-1.51$ & 1.43 & $1.29-1.71$ & 1.42 & $1.31-1.51$ & 1.35 & $1.26-1.44$ & 1.24 & $1.17-1.37$ \\
\hline \multicolumn{11}{|l|}{ Others (Ref.) } \\
\hline \multicolumn{11}{|l|}{ ANC Visit } \\
\hline 1 to 3 & 1.25 & $1.23-1.27$ & 1.19 & $1.18-1.21$ & 1.20 & $1.19-1.22$ & 1.19 & $1.18-1.21$ & 1.24 & $1.21-1.26$ \\
\hline $\begin{array}{l}4 \text { to } 5 \\
>5 \text { (Ref) }\end{array}$ & 0.67 & $.61-.73$ & 0.61 & $.56-.66$ & 0.61 & $.56-.66$ & 0.65 & $.61-.71$ & 0.75 & $.72-.78$ \\
\hline
\end{tabular}

Note: AOR - Adjusted Odd Ratio; CI: Confidence Interval; All odds are significant at P $<0.01$; Ref. - Reference Category. 
years. Women having no education were (AOR: 1.55; $\mathrm{P}<0.001$; CI: 1.49-1.61) times less likely to checked their BP, (AOR: 1.55; P < 0.001; CI: 1.51-1.61) times less likely to tested urine sample and (AOR: 1.57; P $<0.001$; CI: $1.49=1.68$ ) times less likely to take iron supplements. Women reside in the rural area were (AOR: 1.84; $\mathrm{P}<0.001$; CI: 1.79-1.89) times less likely to measured their BP and (AOR: 1.27; P < 0.001; CI: 1.21-1.36) times less likely to tested their blood sample as compared to women who reside in urban area.

\section{Discussion}

Nowadays priority is given to provide the routine check-up treatments, constituent of the ANC treatments is available in many health facilities. ${ }^{35}$ Nonetheless, these are poorly utilized because of ignorance from the part of recipient. Although the subject of routine check-up in ANC in gestational period has produced a large body of literature, ${ }^{36,37}$ there is much less about ANC with only few paper covering the importance of weight and BP measurement ${ }^{17}$ and importance of iron supplement. ${ }^{23}$ Our result showed that EAG states shares a large proportion of women holds the tendency not to receive the routine check-up treatments within the desired ANC. They hardly care about the adverse consequences of apathy to receive the desired check-up. Despite the remarkable progress during last tenure on promotion of infrastructural background and treatments related to maternal health care especially towards ANC, the gap remains in effective disbursement of the treatments. ${ }^{38}$ This study has identified a number of socio-economic and demographic inherited causes of the recipients for non utilisation of ANC treatments. Evidence has found that variability of socio-economic characteristics across the individual level has largely affect on the receiving of desired routine check-up in ANC service care. ${ }^{39}$ A number of studies have documented that socio-economic status of household and maternal educational level are the strong predictor in receiving ANC treatments. ${ }^{3}$ Rural-urban residence of women has significant association with receiving antenatal check-ups during pregnancy. The study result shows rural women were less likely to receive ANC treatments as compared with those women who were living in urban area. This finding is consistent with other studies carried out in India. ${ }^{3}$ Urban women have higher educational knowledge, better health infrastructure in public and private health care facilities, greater awareness regarding their health, on the other hand rural women sometimes get these opportunities. ${ }^{40}$ Delayed access to ANC associated with maternal and foetal mortality and morbidity. ${ }^{41}$ Educational attainment of mother has strong effect on reproductive behaviour and awareness related to health, hygiene, sanitation etc. No education or lesser educational attainment of women largely affect health seeking behaviour of a woman. If women endured deprived in their home and societies, that will definitely affect the health of the mother and new born. Women's education has a significant positive influence on the use of ANC treatments. Women with higher educational attainment are more cautious about the relevance of each routine-check-up in ANC treatments. Consistent with the findings of previous literature, $3,35,39$ our study also revealed that Women with no education or less education usually show the apathy to visit the nearby centre for routine check-up. It seems that they are hardly aware about the adverse consequences of non-receiving of routine care check-up during the gestational period. This finding highlighted the importance of women's education for improvement the behaviour in receiving ANC available in health facilities. Study result implies that higher educated women have better access to health care centre and they have better decision-making ability and they are much aware about negative consequences of not seeking ANC. In case of ANC utilisation, household economic status or the wealth status is likely to be most powerful factor. ${ }^{3}$ The result of this present study revealed that poorest women were less likely to visit ANC and skip regular check-ups as compared to the richest women. Furthermore, our study exhibited that the women who early get married ( $<18$ years of age) were less likely to use ANC treatments as compared to women who get married after 30 years of age. This finding is consistent with previous studies. ${ }^{3}$ The study also indicates that women belonged to socially marginalized category (SC/ST) have significantly lower likelihood of ANC treatments than the women belonged to forward caste. We compare our study results with result from studies which address analogous outcomes. ${ }^{3}$ The women of this marginalized caste were less aware about the desired precaution about do or don'ts during the gestation period. This finding can be demonstrated by the fact that mother belongs to marginalized (SC/ST) category are economically and educationally backward than the forward case. This study has found that the women believed in Muslim religion were less likely to take iron supplements, measured their weight and BP, tested blood and urine samples compared to the other religion. We found that the uptake of iron supplements, weight and BP measurement, test of blood and urine sample during gestational period were associated with socio-economic and demographic background of the study population. Some literature explained BP and weight measurement are very essential during pregnancy as because state of being more or less it causes hypertensive disorder, pre-eclampsia, eclampsia, oedema. ${ }^{12-14}$ Our study result showed that economically poor and less educated women were less likely to measured their weight and BP during gestational period. The study found that young women ( $<18$ years) were less likely to took iron supplements. We compare our result with result from other studies which address similar outcome. ${ }^{30}$ Compared to other study, ${ }^{19,20}$ this study also found that socio-economic and demographic variables play a significant role in receiving iron supplements which control the prevalence of anemia among women. ${ }^{30}$ The study already has showed the result about the eagerness in receiving the desired routine ANC check-up among the women who visited health facilities more than 5 times. Another encouraging finding is that pregnant women who had more ANC visits were receiving more ANC treatments and experiencing lower risk of maternal and neo natal mortality. It seems that women having previous gestational period and birth experience feel more confident to ignore the treatments. Most of the women were availing the ANC treatments at their local health centres; govt. should encourage them to receive these treatments and should pay attention towards empowering women. Routine examination of the pregnant women and foetus may help to reduce adverse pregnancy outcome.

\section{Conclusion}

The findings of this study accentuated the importance of socioeconomic and demographic factors which has a significant impact on the utilisation of ANC treatments. Large expansion in antenatal care coverage in EAG states are still needed. Women empowerment is necessary which starts with proper education, improving working status and by increasing their earning potential that may generate awareness about their reproductive health. ${ }^{42}$ It is possible to improve maternal health outcomes by analysing various social determinants. Degree of awareness of the mother depends upon various socio-economic factors which play the key role to receive the viable and better health care treatments. Socio-economic status of the women in rural areas in EAG states tends to be inferior to that of women residing in the urban areas. Limited access to economic resources, unequal opportunities, less ability to make decision, low social status of women in the society (caste, class, ethnicity), support from marital family and marital relationship, less opportunity for basic education, employment and income, gender norms, religion, woman's age, early marriage, health beliefs, social cohesion, poor guidance, these are the various socio-economic-demographic predictors which determine their perception about ANC treatments. It is possible to improve maternal health outcome through proper routine check-up taken during antenatal care visit. Necessary interventions and awareness at grass root level are very much needed for proper utilisation of routine check-up during gestational period. Some plan and policies also required to empower the rural women of EAG states that may help in implementation of proper utilisation of ANC treatments. 


\section{Ethical approval}

Not Applicable.

\section{Funding information}

This research did not receive any specific grant from funding agencies in the public, commercial, or not-for-profit sectors.

\section{Declaration of competing interest}

None declared.

\section{Declaration of competing interest}

The authors have no conflicts of interest to declare.

\section{Acknowledgements}

All authors have read and approved the final manuscript.

\section{References}

1 WHO. Maternal mortality fact sheet. Matern heal [internet]. 2015, 1-5. Available fromhttp://www.who.int/mediacentre/factsheets/fs348/en/\%5Cnhttp://www.who int/mediacentre/factsheets/fs348/en/index.html ; 2015.

2 JSY- Janani Suraskhya Yojana \& its impact on women. A Case Study on VSS Institute of Medical Sciences Burla, Sambalpur, Odisha JSY- Janani Suraskhya Yojana \& its Impact on Women- A Case Study on VSS Institute of Medical Sciences Burla. Sambalpur: Odis; 2018; (June).

3 Paul P, Chouhan P. Socio-demographic factors influencing utilization of maternal health care treatments in India. Clin Epidemiol Glob Heal [Internet]. 2020;8(3): 666-670. https://doi.org/10.1016/j.cegh.2019.12.023. Available from:.

4 WHO. Trends in mternal mortality: 1990-2013. Estimates by WHO,UNICEF, UNFPA, the world bank and the united nations population division. World Heal Organ [Internet]; 2014:56. Available from http://apps.who.int/iris/bitstream/10665/112 $682 / 2 / 9789241507226$ eng.pdf?ua $=1$.

5 Dutta P. Maternal Health and Maternal Mortality : A Study of Four Selected Districts of Assam. Prafulla Kr. Dutta and Binu Dutta; 2018.

6 Singh RK, Patra S. Differentials in the utilization of antenatal care treatments in EAG states of India. Int Res J Soc Sci [Internet]. 2013;2(11):28-32. Available from www isca.me.

7 Gottfredsdottir H, Steingrímsdóttir P, Björnsdóttir A, Gudmundsdóttir EÝ, Kristjánsdóttir H. Content of antenatal care: does it prepare women for birth? Midwifery [Internet], 39:71-77. Available from https://doi.org/10.1016/j.midw.20 16.05.002; 2016.

8 Pervin J, Moran A, Rahman M, et al. Association of antenatal care with facility delivery and perinatal survival - a population-based study in Bangladesh. BMC Pregnancy Childbirth. 2012;12:1. https://doi.org/10.1186/1471-2393-12-111.

9 Jowett M. Safe motherhood interventions in low-income countries: an economic justification and evidence of cost effectiveness. Health Pol. 2000;53(3):201-228. https://doi.org/10.1016/s0168-8510(00)00089-0. PMID: 10996067.

10 Pallikadavath S, Foss M, Stones RW. Antenatal care: provision and inequality in rural north India. Soc Sci Med. 2004;59(6):1147-1158. https://doi.org/10.1016/j. socscimed.2003.11.045.

11 WHO. Essential Antenatal, Perinatal and Postpartum Care: Training Modules. WHO Reg Off Eur; 2002:1-392.

12 Gupta R, Talukdar B. Frequency and timing of antenatal care visits and its impact on neonatal mortality in EAG states of India. J Neonatal Biol. 2017. https://doi.org/ 10.4172/2167-0897.1000263, 06(03).

13 Chancellor J, Thorp JM. Blood pressure measurement in pregnancy. BJOG An Int $J$ Obstet Gynaecol. 2008;115(9):1076-1077. https://doi.org/10.1111/j.14710528.2008.01755.x.

14 Persson M, Cnattingius S, Wikström AK, Johansson S. Maternal overweight and obesity and risk of pre-eclampsia in women with type 1 diabetes or type 2 diabetes. Diabetologia [Internet]. 2016;59(10):2099-2105. https://doi.org/10.1007/s00125016-4035-z. Available from.

15 Ellison GTH, Holliday M. The use of maternal weight measurements during antenatal care. A national survey of midwifery practice throughout the United Kingdom. $J$ Eval Clin Pract. 1997;3(4):303-317. https://doi.org/10.1046/j.1365-2753.1997.t01-100007.x.

16 July 2020 Bengtson AM, Phillips TK, le Roux SM, et al. High blood pressure at entry into antenatal care and birth outcomes among a cohort of HIV-uninfected women and women living with HIV initiating antiretroviral therapy in South Africa. Pregnancy Hypertens [Internet]. 2021;23:79-86. https://doi.org/10.1016/j. preghy.2020.11.005. Available from.
17 Ngene NC, Moodley J. Blood pressure measurement in pregnancy and in hypertensive disorders of pregnancy: devices, techniques and challenges. Cardiovasc J Afr. 2019;30(2):120-129. https://doi.org/10.5830/CVJA-2018-067.

18 Brown MA, Magee LA, Kenny LC, et al. The hypertensive disorders of pregnancy: ISSHP classification, diagnosis \& management recommendations for international practice. Pregnancy Hypertens. 2018;13(xxxx):291-310. https://doi.org/10.1161/ HYPERTENSIONAHA.117.10803. PMID: 29899139.

19 Wang C, Lin L, Su R, et al. Hemoglobin levels during the first trimester of pregnancy are associated with the risk of gestational diabetes mellitus, pre-eclampsia and preterm birth in Chinese women: a retrospective study. BMC Pregnancy Childbirth. 2018;18(1):1-11. https://doi.org/10.1186/s12884-018-1800-7.

20 Sey-Sawo J, Tunkara-Bah H. Iron deficiency anemia in Pregnancy : the fate of the mother and the unborn child in the Gambia. Int J Innov Res Adv Stud. 2016;3(12):1-6.

21 Urassa DP, Carlstedt A, Nyström L, Massawe SN, Lindmark G. Eclampsia in Dar es Salaam, Tanzania - incidence, outcome, and the role of antenatal care. Acta Obstet Gynecol Scand. 2006;85(5):571-578. https://doi.org/10.1080/ 00016340600604880 . PMID: 16752236.

22 Narice B, Martinez G, Amabebe E, Rehman I, Anumba D. Raman spectroscopic analysis of cervicovaginal fluid as a predictive tool for spontaneous preterm birth. BJOG An Int J Obstet Gynaecol. 2018;125(S2):108-109.

23 Alwan NA, Cade JE, McArdle HJ, Greenwood DC, Hayes HE, Simpson NAB. Maternal iron status in early pregnancy and birth outcomes: insights from the Baby's Vascular health and Iron in Pregnancy study. Br J Nutr. 2015;113(12):1985-1992. https://doi. org/10.1017/S0007114515001166.

25 Mora JO, Nestel PS. Improving prenatal nutrition in developing countries: strategies, prospects, and challenges. Am J Clin Nutr. 2000;71(5 suppl L). https://doi.org/ 10.1093/ajen/71.5.1353s. Available from.

26 Zuhair M, Roy RB. Socioeconomic determinants of the utilization of antenatal care and child vaccination in India. Asia Pac J Publ Health. 2017;29(8):649-659. https:// doi.org/10.1177/1010539517747071.

27 Acharya SK. Demographic and socio-economic factors affecting antenatal care treatments utilization in Nepal. J Dev Adm Stud. 2018;24(1-2):71-87. https://doi. org/10.3126/jodas.v24i1-2.19668.

28 Office of registrar general I. Annual health survey (AHS) in 8 EAG states and Assam release of AHS bulletin: 2010-11. Gov India [Internet]; 2011:8-9. Available from htt p://censusindia.gov.in/vital_statistics/AHSBulletins/files/AHSpr.pdf.

29 Kakati R, Barua K, Borah M. Factors associated with the utilization of antenatal care treatments in rural areas of Assam, India. Int J Community Med Public Heal. 2016;3 (10):2799-2805. https://doi.org/10.18203/2394-6040.ijcmph20163364. Available from.

30 Singh RK. Lifestyle behavior affecting prevalence of anemia among women in EAG states, India. J Public Health. 2012;21(3):279-288. https://doi.org/10.1007/s10389012-0548-1.

31 Maternal \& Child Mortality and Total Fertility Rates Sample Registration System ( SRS ) Office of Registrar General , India. 2011;(July).

32 World Health Organization. World Health Statistics: Health-Related Millennium Development Goals. WHO Libr Cat Data; 2015.

33 Nambile Cumber S. Importance of antenatal care treatments to pregnant women at the Buea regional hospital Cameroon. J Fam Med Health Care. 2016;2(4):23. https:// doi.org/10.11648/j.jfmhc.20160204.11.

34 Konlan KD, Saah JA, Amoah RM, et al. Factors influencing the utilization of Focused antenatal care treatments during pregnancy, a study among postnatal women in a tertiary healthcare facility, Ghana. Nurs Open. 2020;7(6):1822-1832. https://doi. org/10.1002/nop2.569. Available from.

35 Nguyen HTH, Hatt L, Islam M, et al. Encouraging maternal health service utilization: an evaluation of the Bangladesh voucher program. Soc Sci Med [Internet]. 2012;74 (7):989-996. https://doi.org/10.1016/j.socscimed.2011.11.030. Available from.

36 Laksono AD, Rukmini R, Wulandari RD. Regional disparities in antenatal care utilization in Indonesia. PLoS One [Internet. 2020;15(2):1-13. https://doi.org/ 10.1371/journal.pone.0224006. Available from.

37 Nigenda G, Langer A, Kuchaisit C, et al. Womens' opinions on antenatal care in developing countries: results of a study in Cuba, Thailand, Saudi Arabia and Argentina. BMC Publ Health. 2003;3:1. https://doi.org/10.1186/1471-2458-3-17.

38 Ghosh A, Ghosh R. Maternal health care in India: a reflection of 10 years of National Health Mission on the Indian maternal health scenario. Sex Reprod Healthc [Internet]. 2020. December 2019;25, 100530. https://doi.org/10.1016/j.srhc.2020.100530. Available from

39 Oladapo OT, Osiberu MO. Do sociodemographic characteristics of pregnant women determine their perception of antenatal care quality? Matern Child Health J. 2009;13 (4):505-511. https://doi.org/10.1007/s10995-008-0389-2.

40 Singh L, Rai RK, Singh PK. Assessing the utilization of maternal and child health care among married adolescent women: evidence from India. J Biosoc Sci. 2012;44(1): 1-26. https://doi.org/10.1017/S0021932011000472. Epub 2011 Sep 21. PMID: 21933465.

41 Haddrill R, Jones GL, Mitchell CA, Anumba DOC. Understanding delayed access to antenatal care: a qualitative interview study. BMC Pregnancy Childbirth. 2014;14(1): 1-14. https://doi.org/10.1186/1471-2393-14-207.

42 Chopra I, Juneja SK, Sharma S. Effect of maternal education on antenatal care utilization, maternal and perinatal outcome in a tertiary care hospital. Int $J$ Reprod Contraception, Obstet Gynecol. 2018;8(1):247. https://doi.org/10.18203/2320-1770. ijrcog20185433. 